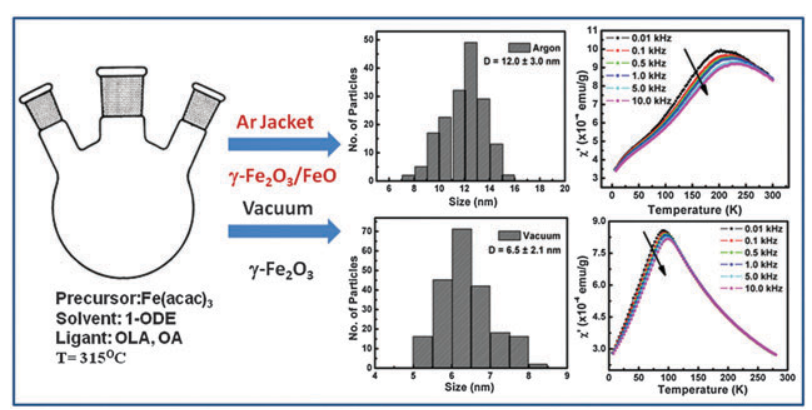

\author{
Synthesis, phase composition, Mössbauer and \\ magnetic characterization of iron oxide \\ nanoparticles \\ Sarveena, J. M. Vargas, D. K. Shukla, C. T. Meneses, \\ P. Mendoza Zélis, M. Singh and S. K. Sharma* \\ Synthesis methods clearly show the effect of argon and \\ vacuum environments on the structure-property \\ relationship of iron oxide nanoparticles. A clear \\ contradiction is observed from the results of static and \\ dynamic magnetization for both samples.
}

Please check this proof carefully. Our staff will not read it in detail after you have returned it.

Translation errors between word-processor files and typesetting systems can occur so the whole proof needs to be read. Please pay particular attention to: tabulated material; equations; numerical data; figures and graphics; and references. If you have not already indicated the corresponding author(s) please mark their name(s) with an asterisk. Please e-mail a list of corrections or the PDF with electronic notes attached - do not change the text within the PDF file or send a revised manuscript. Corrections at this stage should be minor and not involve extensive changes. All corrections must be sent at the same time.

Please bear in mind that minor layout improvements, e.g. in line breaking, table widths and graphic placement, are routinely applied to the final version.

Please note that, in the typefaces we use, an italic vee looks like this: $v$, and a Greek nu looks like this: $\nu$.

We will publish articles on the web as soon as possible after receiving your corrections; no late corrections will be made.

Please return your final corrections, where possible within $\mathbf{4 8}$ hours of receipt, by e-mail to: pccparsc.org 


\section{Queries for the attention of the authors}

Journal: PCCP

Paper: c5cp07698f

Title: Synthesis, phase composition, Mössbauer and magnetic characterization of iron oxide nanoparticles

Editor's queries are marked on your proof like this $Q 1, Q_{2}$, etc. and for your convenience line numbers are indicated like this $5,10,15, \ldots$

Please ensure that all queries are answered when returning your proof corrections so that publication of your article is not delayed.

\begin{tabular}{|l|l|l|}
\hline $\begin{array}{c}\text { Query } \\
\text { reference }\end{array}$ & \multicolumn{1}{|c|}{ Query } & Remarks \\
\hline Q1 & $\begin{array}{l}\text { For your information: You can cite this article before you } \\
\text { receive notification of the page numbers by using the } \\
\text { following format: (authors), Phys. Chem. Chem. Phys., } \\
\text { (year), DOI: 10.1039/c5cp07698f. }\end{array}$ & \\
\hline Q2 & $\begin{array}{l}\text { Please carefully check the spelling of all author names. } \\
\text { This is important for the correct indexing and future } \\
\text { citation of your article. No late corrections can be made. }\end{array}$ & \\
\hline Q3 & $\begin{array}{l}\text { The sentence beginning "Recently, much attention..." } \\
\text { has been altered for clarity, please check that the meaning } \\
\text { is correct. }\end{array}$ & \\
\hline Q4 & $\begin{array}{l}\text { A citation to Fig. 6 has been added here, please check that } \\
\text { the placement of this citation is suitable. If the location is } \\
\text { not suitable, please indicate where in the text the citation } \\
\text { should be inserted. }\end{array}$ & \\
\hline Q5 & $\begin{array}{l}\text { The caption to Fig. 8 refers to parts (a) and (b) but the } \\
\text { figure as supplied does not contain parts labelled (a) and } \\
\text { (b). Would you like to modify the caption or resupply the } \\
\text { artwork (preferably as a TIF file at 600 dots per inch)? }\end{array}$ & \\
\hline
\end{tabular}




\title{
Synthesis, phase composition, Mössbauer and magnetic characterization of iron oxide nanoparticles $\dagger$
}

\author{
Sarveena, $^{a}$ J. M. Vargas, ${ }^{b}$ D. K. Shukla, ${ }^{C}$ C. T. Meneses, ${ }^{d}$ P. Mendoza Zélis, ${ }^{e}$ \\ M. Singh ${ }^{a}$ and S. K. Sharma*af
}

The present work describes the synthesis of iron oxide nanoparticles by thermal decomposition of Fe-precursors in argon and vacuum environments with control over particle size distribution, phase composition and the resulting magnetic properties. The Rietveld analysis of $\mathrm{X}$-ray diffraction data revealed the crystallinity as well the single-phase of $\gamma-\mathrm{Fe}_{2} \mathrm{O}_{3}$ nanoparticles prepared under vacuum, whereas the argon environment leads to the formation of multi-phase composition of $\gamma-\mathrm{Fe}_{2} \mathrm{O}_{3} / \mathrm{Fe}_{3} \mathrm{O}_{4}$ (90\%) and wustite (10\%). Synchrotron X-ray absorption near edge structure (XANES) indicates that the predominant phase in both the samples is $\gamma-\mathrm{Fe}_{2} \mathrm{O}_{3}$, which is subsequently verified from the Mössbauer

Received 14th December 2015 Accepted 22nd February 2016

DOI: $10.1039 / c 5 c p 07698 f$

www.rsc.org/pccp spectra. DC magnetic measurements indicate behavior typical of a superparamagnetic system validated by Mössbauer analysis. However, further investigation of ac susceptibility by typical Néel-Arrhenius and Vogel Fulcher magnetic models suggests an influence of interparticle interactions on the overall magnetic behavior of the system.

\section{Introduction}

Magnetic iron oxide based nanoparticles have become the center of huge attraction among the scientific community because of their increasing number of applications in various

QQ3 fields. ${ }^{1}$ Recently, much attention has been paid towards the superparamagnetic $\mathrm{Fe}_{3} \mathrm{O}_{4}$ nanoparticles not only because of their complex magnetic behavior, but also their suitability in various biological applications. ${ }^{2,3}$ However, one of the most significant tasks in the synthesis of iron oxide nanoparticles is

40 the control over the phase purity, which hinders distinguishing between the pure magnetite and the maghemite phase, as their crystal structures are very close. Furthermore, $\mathrm{Fe}_{3} \mathrm{O}_{4}$ has been predicted to be half metallic at $300 \mathrm{~K}$ and hence is expected to produce $100 \%$ spin polarization of an electric current passing

${ }^{a}$ Department of Physics, H. P. University, Shimla 171005, HP, India

${ }^{b}$ Centro Atomico Bariloche (CNEA), Instituto Balseiro (U. N. Cuyo) and Conicet, 8400 San Carlos de Bariloche, Río Negro, Argentina

${ }^{c}$ UGC DAE Consortium for Scientific Research, Indore 452001, India

$50{ }^{d}$ Núcleo de Pós-Graduação em Física, Campus Prof. José Aluísio de Campos, UFS, 49100-000 São Cristóvão, SE, Brazil

${ }^{e}$ Instituto de Física de La Plata (IFLP-CONICET), Universidade Nacional de La Plata (UNLP), c.c. 67, 1900 La Plata, Argentina

${ }^{f}$ Universidade Federal do Maranhão, Departamento de Física, 65080-805, São Luis, MA, Brazil. E-mail: surender76@gmail.com

$55 \dagger$ Electronic supplementary information (ESI) available. See DOI: 10.1039/ c5cp07698f through. ${ }^{4}$ Thin films of $\mathrm{Fe}_{3} \mathrm{O}_{4}$ nanoparticles have been shown to display very interesting magneto-resistance values in comparison to thin films prepared by physical methods and are expected to lead to the development of magneto-electronic devices with enhanced magneto-transport properties. ${ }^{5}$ Besides these, at the nanoscale regime, $\mathrm{Fe}^{2+}$, in $\mathrm{Fe}_{3} \mathrm{O}_{4}\left(\left[\mathrm{Fe}^{3+}\right]_{\mathrm{A}}\left[\mathrm{Fe}^{3+-}\right.\right.$ $\left.\mathrm{Fe}^{2+}\right]_{\mathrm{B}} \mathrm{O}_{4}$ ) with $\mathrm{A}$ - and $\mathrm{B}$ the so called tetrahedral and octahedral sites, becomes very sensitive to the oxidation state hence may change the nanoparticle composition and properties, particularly at the surface. Therefore, the reproducibility of iron oxide nanoparticles (IONPs) with desired controlled phase compositions and magnetic properties is a challenging task, and equally important from the application point of view. With regard to this, researchers are trying to optimize the synthesis conditions leading to reproducible sizes and compositions and hence the desired magnetic properties of nanoparticles. Moreover, a very important issue is to better understand the synthesis recipe in order to have a better control over the size/shape distribution and hence to establish a good relation with size and structure dependent magnetic properties.

Different synthesis strategies have been found in the literature for obtaining iron oxide nanoparticles with well-defined size/shape and phase composition. ${ }^{6-8}$ However, among them, synthesis by the thermal decomposition of a metal precursor in a high boiling solvent appears to be the most interesting because it permits a good control over the size and the morphology of the nanoparticles. ${ }^{9,10}$ This is mainly due to the 
1 fact that the synthesis recipe is simple with one iron precursor, one type of organic ligand, and a high boiling point organic solvent. However at the same time, despite a lot of optimized synthesis reports, the influences of some synthesis parameters remain unclear such as the inert atmosphere in the three-neck flask during refluxing. ${ }^{11}$ As observed with other synthesis routes, the magnetic moment of superparamagnetic iron oxide is much lower than that of bulk phase magnetite or its fully oxidized form: maghemite $\left(\gamma-\mathrm{Fe}_{2} \mathrm{O}_{3}\right)$. The spin canted layer at

10 the nanoparticle surface is reported as the main cause of it, but at the same time the presence of a large number of defects and/ or of a spin canting in volume is also not ignored. Therefore, along with the compositional control there is a current requirement for the fine structural and magnetic characterization as a 15 function of the nanoparticle size to establish size-dependent magnetic properties.

In the present paper, we have performed synthesis either in an inert atmosphere or in a controlled vacuum in a three-neck round bottom flask to achieve the phase purity of iron oxide nanoparticles (IONPs) and studied their magnetic properties. First synthesis has been performed in an argon atmosphere, whereas the second one carried out in a vacuum $\left(10^{-2}\right.$ mbar $)$ through a simple rotary pump. The rest of the synthesis conditions were exactly same in both the cases. These nanoparticles have been investigated structurally and magnetically, through X-ray diffraction (XRD), high resolution transmission electron microscopy (HR-TEM), soft X-ray absorption spectroscopy (SXAS), Mössbauer spectroscopy (MS) at $300 \mathrm{~K}$, and magnetic measurements in both ac and dc modes in order to correlate the structural and hence magnetic properties.

\section{Experimental section}

\section{Synthesis of iron oxide nanoparticles}

The size of iron oxide nanoparticles (IONPs) has been controlled during synthesis by performing it either in an inert atmosphere or a vacuum using a standard inert gas/vacuum 40 manifold system commonly known as the Schlenk line. Synthesis was carried out in a standard three neck round bottom flask using thermal decomposition of $\mathrm{Fe}(\mathrm{acac})_{3}(2.0 \mathrm{mmol})$ in the presence of 1-octadecene $(20 \mathrm{ml})$ and the surfactants oleic acid $(8 \mathrm{ml})$ and oleylamine $(12 \mathrm{ml})$ either in pure argon flux or

45 in a vacuum. The above mixture was gently heated at $120^{\circ} \mathrm{C}$ for 60 minutes under continuous argon or vacuum with intermediate stirring. The above mixture was slowly heated up to a final temperature of $315{ }^{\circ} \mathrm{C}$ for 60 minutes with a heating rate of $\sim 6{ }^{\circ} \mathrm{C} \min ^{-1}$. The solution was then cooled down to room temperature under normal conditions and the nanoparticles were washed adding excess of ethanol and centrifuged at 4500 $\mathrm{rpm}$ for 20 minutes. This procedure was repeated 3-4 times by dispersing the NPs in a non-polar solvent, adding excess of ethanol and centrifugation was carried out. Finally, the nanoparticles were dispersed in toluene (concentration of $0.05 \mathrm{~g} \mathrm{ml}^{-1}$ ) for a long-term storage with 2-3 drops of oleylamine.

\section{Characterization}

The particle diameter and its distribution were measured by means of HRTEM (200 keV JEM 2010 microscope) by drying a toluene dispersion of the nanoparticles on a carbon coated copper grid. The structure was determined by X-ray diffraction (XRD) (X'PERT Powder PANalytical) with $\mathrm{Cu} \mathrm{K}_{\alpha}$ radiation. The Rietveld refinement analysis of XRD patterns was performed using the DBWSTools2.3 refinement program. ${ }^{12,13}$ A pseudoVoigt function was selected to fit the observed peak profiles of the identified crystalline phase. The average crystallite size was calculated using all Bragg reflections by the Scherrer equation. The full width at half maximum (FWHM) of each peak was corrected with a standard sample of $\mathrm{LaB}_{6}$. Further details of crystallite size calculation and profile function can be found in ref. 14 and 15. The Mössbauer spectrum was also measured employing a homemade Mössbauer spectrometer working in a constant acceleration mode and equipped with a ${ }^{57} \mathrm{Co}(\mathrm{Rh})$ source with an activity of $50 \mathrm{mCi}$. The isomer shift values were referred to $\alpha$-Fe foil at room temperature. X-ray absorption spectroscopy (XAS) measurements across $\mathrm{Fe}_{\mathrm{L}, 2}$ and $\mathrm{O}$ K-edge were performed at the SXAS beamline (BL-01) of the INDUS 2 synchrotron source at RRCAT, Indore. XAS data were collected in total electron yield (TEY) mode at RT under ultra-high vacuum conditions. Pre and post edge correction of normalized XAS data were performed using Athena. The magnetic properties were measured on a dried powder sample using a SQUID (Quantum Design) magnetometer with fields up to $40 \mathrm{kOe}$ and temperatures from 5-300 K. The ZFC and FC measurements were carried out as follows: the sample was first cooled down from $300 \mathrm{~K}$ to $5 \mathrm{~K}$ in a zero magnetic field, then a static magnetic field ( $H=50 \mathrm{Oe}$ ) was applied and $M_{\mathrm{ZFC}}$ was measured during warming up from $5 \mathrm{~K}$ to $300 \mathrm{~K}$; finally the sample was cooled down to $5 \mathrm{~K}$ under the same field and $M_{\mathrm{FC}}$ was measured during the cooling cycle. The real and imaginary parts of the ac magnetic susceptibility were measured at frequencies between $10 \mathrm{~Hz}<f<10 \mathrm{kHz}$ in an external ac field amplitude of 10 Oe on a commercial Physical Property Measurement System (PPMS) in the temperature range 5-300 K.

\section{Results and discussion}

The phase purity and composition have been checked using the powder XRD method and Rietveld analysis. Fig. 1 shows the powder XRD patterns for the two samples of iron oxide prepared under argon flux and vacuum. By analyzing the XRD patterns, it is confirmed that the brownish black powder obtained in the case of a sample prepared under argon consisted mainly of the maghemite/magnetite $\left(\gamma-\mathrm{Fe}_{2} \mathrm{O}_{3} / \mathrm{Fe}_{3} \mathrm{O}_{4}\right)$ $(90(2) \%)$ as well as the wustiteFeO phase $(10(4) \%)$, whereas the sample prepared under vacuum confirmed the formation of maghemite $\left(\gamma-\mathrm{Fe}_{2} \mathrm{O}_{3}\right)$ as the major phase. Here we would like to mention that the most intense peaks of the $\mathrm{FeO}$ phase $(\{111\}$, $2 \theta \cong 36.5^{\circ} ;\{200\}, 2 \theta \cong 42.8^{\circ} ;\{220\}, 2 \theta \cong 62.1^{\circ}$ ) are overlapped with the Bragg peaks $\{222\},\{004\}$ and $\{044\}$ of the $\gamma-\mathrm{Fe}_{2} \mathrm{O}_{3} / \mathrm{Fe}_{3} \mathrm{O}_{4}$ phase. Thus, these peaks cannot be pulled apart among the 

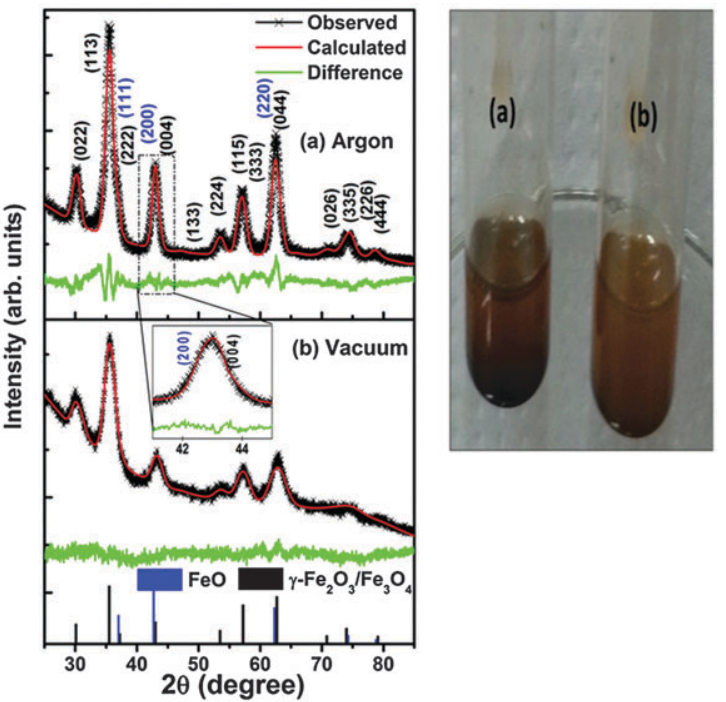

Fig. 1 (left panel) X-ray diffraction patterns for iron oxide nanoparticle (IONP) samples prepared under argon or vacuum conditions, (right panel) $\gamma-\mathrm{Fe}_{2} \mathrm{O}_{3} / \mathrm{Fe}_{3} \mathrm{O}_{4}$ (in black) and $\mathrm{FeO}$ (in blue). The inset shows the planes (200) and (004) of $\mathrm{FeO}$ and $\gamma-\mathrm{Fe}_{2} \mathrm{O}_{3} / \mathrm{Fe}_{3} \mathrm{O}_{4}$ phases, respectively.

However, the two major oxides of Fe namely maghemite $\left(\gamma-\mathrm{Fe}_{2} \mathrm{O}_{3}\right)$ and magnetite $\left(\mathrm{Fe}_{3} \mathrm{O}_{4}\right)$ are both cubic inverse spinels and structurally very similar to each other.

Further from the XRD patterns, it is evident that the reflection lines are quite broad, suggesting the miniaturization of the powder crystallites into nanosized particles. Using the full width half the maximum (FWHM) and positions of the most intense peaks extracted from Rietveld refinement we have estimated the particle sizes. The average particle sizes were calculated without considering the possible contributions of crystal strain to the observed broadening by using Scherrer's equation;

$$
D=\frac{k \lambda}{B \cos \theta}
$$

40 where $D$ is the average particle size, $k$ is a shape factor for which a value of 0.9 is used and $\lambda$ is the wavelength of the incident X-ray. Here $B=\left(B_{\mathrm{M}}{ }^{2}-B_{\mathrm{S}}{ }^{2}\right)^{1 / 2}$, where $B_{\mathrm{M}}$ is the FWHM of the XRD peak and $B_{\mathrm{S}}$ is the standard instrumental broadening. The average particle size calculated using eqn (1) are $7.4 \mathrm{~nm}$ and $45 \quad 5.1 \mathrm{~nm}$ for argon and vacuum condition, respectively and consists of nanosized particles for which superparamagnetic effects should be expected.

Fig. 2 highlights the micro-structural features of both iron oxide nanoparticles. Here Fig. 2a and e display the representaargon atmosphere as well as in a vacuum along with their high resolution images, respectively.

In Fig. $2 \mathrm{~b}$ and e the faceted-spherical shape can be clearly observed and we observed that there is no agglomeration among the particles owing to the organic capping on their surfaces. Evidently the nanoparticles obtained under an argon
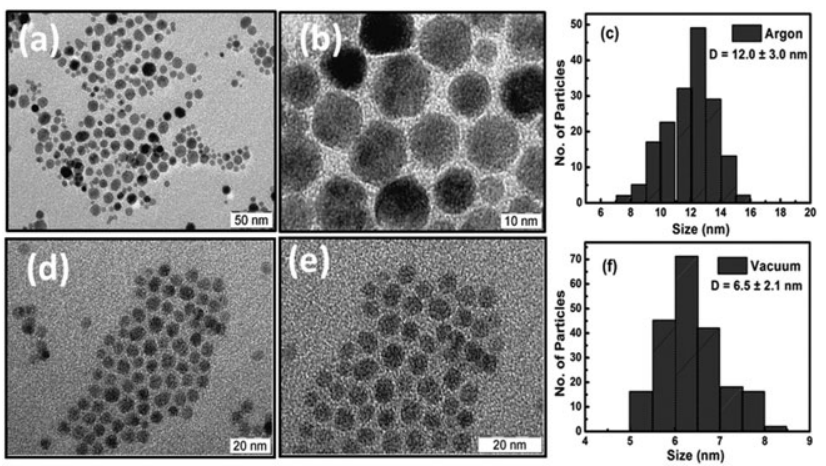

Fig. 2 TEM images of iron oxide samples prepared in an argon atmosphere $(a-c)$ and in a vacuum $(d-f)$ along with their particle size distribution histograms

atmosphere are more polydisperse as compared to the ones obtained under vacuum condition (Fig. 2). The reason for this remarkable difference in the aforementioned synthesis paths is not clearly understood yet. The detailed explanation of this issue is beyond the scope of this paper, where more systematic experiments at different vacuum levels are required as the boiling point of 1-octadecene changes as a function of atmospheric pressure. However, the vacuum path promotes a narrower single-step process of nucleation and growth of nanoparticles, which means narrow particle size distribution. It is observed in the images (see Fig. 2a and b) that most of the particles show an inner contrast variation suggesting probably the existence of two phase structure consisting of $\gamma-\mathrm{Fe}_{2} \mathrm{O}_{3} / \mathrm{Fe}_{3} \mathrm{O}_{4}$ and $\mathrm{FeO}$. Moreover, we have also seen the absence of such a contrast variation in most of the particles which could be explained by the complete oxidation of those particles during the washing process or thereafter. Selected area electron diffraction (SAED) was also performed in order to see the crystallinity of the samples and the corresponding patterns are shown in ESI, $\uparrow$ Fig. S1. However, it was not possible to quantify the interplanar distance of each electron diffraction ring, but the observed results hint towards a better crystallinity of both the samples.

It is not very straightforward to distinguish between two forms of iron oxide namely maghemite $\left(\gamma-\mathrm{Fe}_{2} \mathrm{O}_{3}\right)$ and magnetite $\left(\mathrm{Fe}_{3} \mathrm{O}_{4}\right)$ from the XRD patterns, therefore X-ray absorption spectroscopy has been used to understand the oxidation states of Fe for these two samples. Fig. 3 shows X-ray absorption near edge spectra (XANES) across $\mathrm{Fe}_{\mathrm{L}_{3,2}}$ edges (Fig. 3a) and $\mathrm{O} \mathrm{K}$ edges (Fig. 3a) for iron oxide nanoparticles along with reference compound $\gamma-\mathrm{Fe}_{2} \mathrm{O}_{3}$.

$\mathrm{Fe}_{3,2}$ edge XANES probes the unoccupied 3d states of Fe via electron transitions from spin orbit split levels $2 \mathrm{p}_{3 / 2}$ to $3 \mathrm{~d}$ ( $\mathrm{L}_{3}$ edge) and $2 \mathrm{p}_{1 / 2}$ to $3 \mathrm{~d}\left(\mathrm{~L}_{2}\right.$ edge). Due to crystal field splitting $L_{3}$ and $L_{2}$ edges are further split into $t_{2 g}$ and e levels (marked for $L_{3}$ edge). A careful observation of $L_{3}$ edge shows that the $t_{2 g}$ feature is suppressed in nanoparticles; suppressed relatively more in the nanoparticles prepared in a vacuum. A weaker (stronger) $t_{2 g}$ feature in the $L_{3}$ edge of $F e$ is indicative of less (more) un-occupancy in the $\mathrm{t}_{2 \mathrm{~g}}$ state, which is the case for $\mathrm{Fe}^{2+}$ 
Fig. 3 (a) Fe $\mathrm{L}_{3,2}$ edges and (b) $O \mathrm{~K}$ edges XANES recorded in TEY mode for iron oxide nanoparticles prepared in an argon atmosphere, and under vacuum conditions along with reference compound $\gamma-\mathrm{Fe}_{2} \mathrm{O}_{3}$

$\left(\mathrm{Fe}^{3+}\right)$, so supporting the situations for $\mathrm{Fe}_{3} \mathrm{O}_{4}: \mathrm{Fe}^{2+} \& \mathrm{Fe}^{3+}$ $\left(\gamma-\mathrm{Fe}_{2} \mathrm{O}_{3}\right.$ : only $\left.\mathrm{Fe}^{3+}\right)$. A slightly increased $\mathrm{t}_{2 \mathrm{~g}}$ feature for nanoparticles prepared in argon is indicative of increased $\mathrm{Fe}^{3+}$ content (indicative of the presence of $\gamma-\mathrm{Fe}_{2} \mathrm{O}_{3}$ ). Oxygen $\mathrm{K}$ edge features here are clearer for disentangling the $\gamma-\mathrm{Fe}_{2} \mathrm{O}_{3}$ and $\mathrm{Fe}_{3} \mathrm{O}_{4}$ phases. Features at around $530 \mathrm{eV}$ in XANES at the $\mathrm{O}$ K edge probe the transition of $\mathrm{O} 1 \mathrm{~s}$ electrons into $\mathrm{O} 2 \mathrm{p}$ states (hybridized with Fe 3d states, split into $\mathrm{Fe}_{2 \mathrm{~g}}$ and $\mathrm{e}_{\mathrm{g}}$ states). ${ }^{16}$ While features above 535 are $\mathrm{O} 1 \mathrm{1}$ electrons' transitions into O 2p states hybridized with $\mathrm{Fe}(4 \mathrm{~s}, 4 \mathrm{p})$ states. ${ }^{16}$ Intensity ratios of $t_{2 g}$ and $e_{g}$ features $(\sim 530 \mathrm{eV})$ for vacuum nanoparticles mimic the $\mathrm{O} \mathrm{K}$ edge feature of $\mathrm{Fe}_{3} \mathrm{O}_{4}$, reported in the literature. ${ }^{17}$ Features $t_{2 g}$ and eg in argon nanoparticles show deviation from the $\mathrm{Fe}_{3} \mathrm{O}_{4}$ feature and the tendency of $\gamma-\mathrm{Fe}_{2} \mathrm{O}_{3}$ features, due to the presence of a slight admixture of $\gamma-\mathrm{Fe}_{2} \mathrm{O}_{3}$.

${ }^{57} \mathrm{Fe}$ Mössbauer spectroscopy is a powerful tool to characterize IONPs undergoing superparamagnetic (SPM) relaxation. Fig. 4 shows the Mössbauer spectra recorded for the two samples at $300 \mathrm{~K}$, while the values of the Mössbauer hyperfine parameters, derived from the fitting of the recorded Mössbauer spectra, are listed in Table 1. Here, the dots in Fig. 4 represent the experimental data and solid lines through data points are least squares fittings. The $300 \mathrm{~K}$ Mössbauer spectrum of the both samples shows relaxation effects owing to the nanometric particle size. In both samples a doublet contribution corresponding to superparamagnetism is recognized. The sample
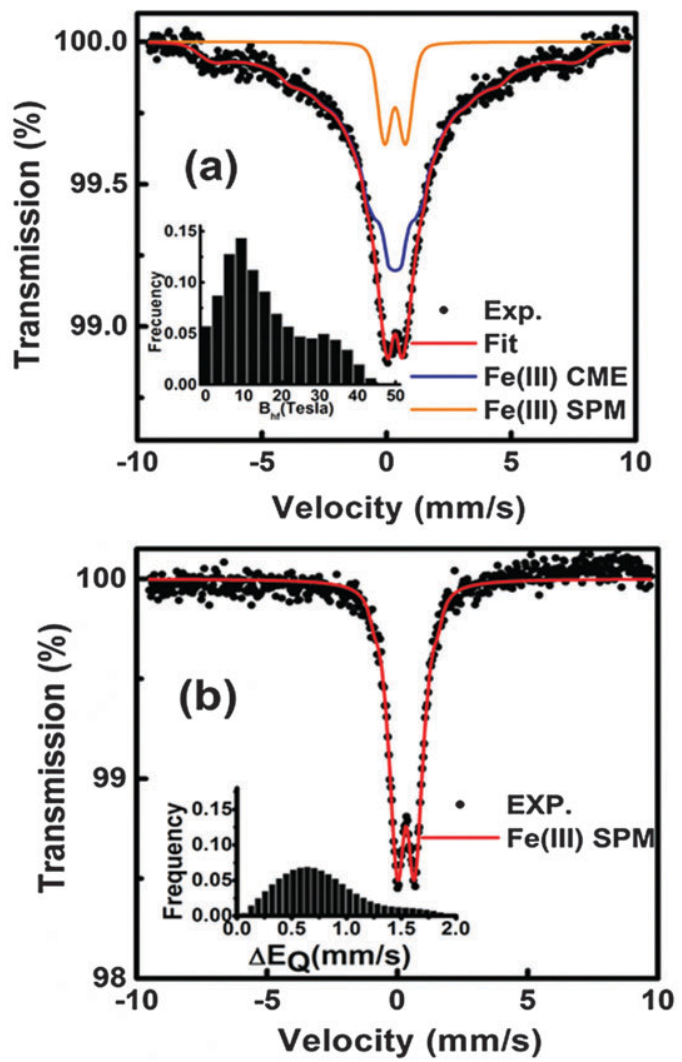

15

Fig. 4 Mössbauer spectra of the iron oxide nanoparticles at $300 \mathrm{~K}$ prepared in (a) an argon atmosphere, and (b) under vacuum conditions.

prepared in an argon atmosphere, according to its bigger size, also presents a distributed sextet component characteristic of collective magnetic excitation (CME) phenomena. In this case, the fitting was made using one distribution of hyperfine fields and one doublet. The corresponding values of the Mössbauer hyperfine parameters (see Table 1), in particular the isomer shift values, are typical of a high-spin Fe(III) atom in iron(III) oxides. ${ }^{18}$ There is no indication of the presence of the $\mathrm{Fe}^{2+}$ valence state, which confirms that both samples are solely of the $\mathrm{Fe}_{2} \mathrm{O}_{3}$ origin.

This is quite expected in connection with their nano size with a large surface-to-volume ratio securing their complete oxidation during the synthesis. On the timescale of the Mössbauer spectroscopy, the nanoparticles of both samples present thermally induced reversal of their magnetization direction at $300 \mathrm{~K}$. Here, the doublet component belongs to the nanoparticles with thermally fluctuating super spins having relaxation times much smaller than the characteristic measurement time $\left(\tau_{\mathrm{m}}\right)$ of the Mössbauer spectroscopy, i.e. superparamagnetic relaxation; while the presence of a distributed sextet component corresponds to nanoparticles whose super spin thermally fluctuates between the energetically favored orientations with a relaxation time close to $\tau_{\mathrm{m}}$, i.e. collective magnetic excitations. These two samples would show superparamagnetic features in their dc magnetization measurements at $300 \mathrm{~K}$, however, this will be largely driven by finite-size and surface 
Table 1 Values of the Mössbauer hyperfine parameters, derived from the Mössbauer spectrum recorded at $300 \mathrm{~K}$, where isomer shift (IS), quadrupole splitting $(Q S)$, mean magnetic hyperfine field $\left(B_{\mathrm{hf}}\right)$, relative area \% and assignments of individual spectral components are shown

\begin{tabular}{|c|c|c|c|c|c|}
\hline Sample & Component (area) & IS $\left( \pm 0.01 \mathrm{~mm} \mathrm{~s}^{-1}\right)$ & $\mathrm{QS}\left( \pm 0.01 \mathrm{~mm} \mathrm{~s}^{-1}\right)$ & $B_{\mathrm{hf}}( \pm 0.1 T)$ & Assignment \\
\hline \multirow[t]{2}{*}{ Argon } & Doublet (14\%) & 0.34 & 0.84 & - & $\mathrm{Fe}^{3+}$ SPM component \\
\hline & Sextet $(86 \%)$ & 0.34 & - & 15.8 & $\mathrm{Fe}^{3+} \mathrm{CME}$ component \\
\hline Vacuum & Doublet (100\%) & 0.33 & 0.85 & - & $\mathrm{Fe}^{3+}$ SPM component \\
\hline
\end{tabular}

effects which can be depicted by a smaller saturation moment or the lack of full saturation etc.

Further, we have performed magnetization $(M)$ measurements as a function of both temperature $(T)$ as well as applied magnetic field $(H)$. Fig. 5 shows the variation of $M$ as a function of $T$ in the range $5-300 \mathrm{~K}$ in an external magnetic field of $50 \mathrm{Oe}$ recorded in zero-field-cooled (ZFC) and field-cooled (FC) modes for both the samples.

In the ZFC curves, a characteristic superparamagnetic (SPM) peak confirms the nanoscale nature of iron oxide particles. In addition, the separation of ZFC and FC curves at a certain irreversibility $T_{\mathrm{IRR}}$ temperature is one of the characteristic features of SPM. The maximum observed in the ZFC curves $T_{\text {MEAN }}$ (related to the mean blocking temperature $T_{\mathrm{B}}$ ) is slightly lower than $T_{\mathrm{IRR}}$. Such behavior indicates a particle size distribution, whereas a fraction of the largest particles already freezes at $T_{\mathrm{IRR}}$, the majority fraction of the nanoparticles is being blocked at around $T_{\mathrm{MEAN}}$. It is evident from Fig. $5 \mathrm{a}$ that there is a sharp maximum in the $M_{\mathrm{ZFC}}$ curve $(\sim 53 \mathrm{~K}$ for the sample prepared under vacuum as compared to the others
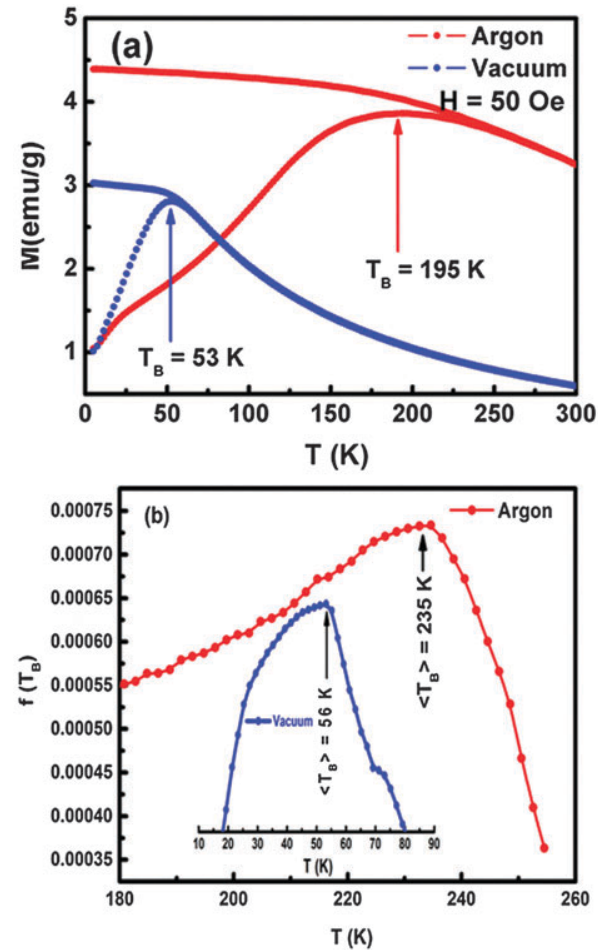

Fig. 5 (a) Zero-field-cooled (ZFC) and field-cooled (FC) curves for the iron oxide sample, (b) the mean blocking temperature calculated from the distribution of blocking temperatures.

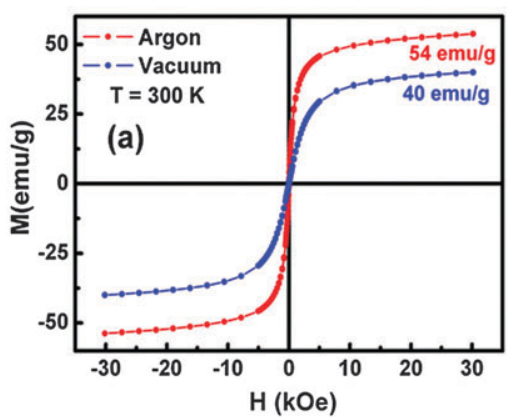

10

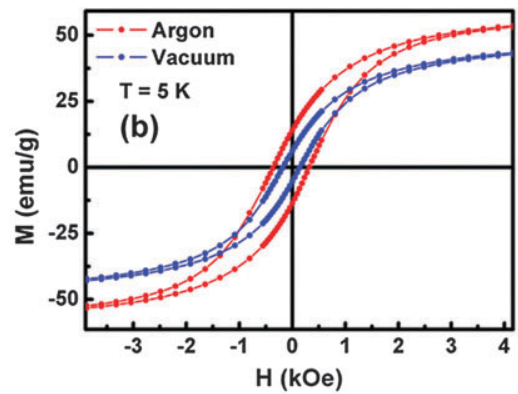

Fig. $6 \mathrm{MH}$ curves of the iron oxide samples at (a) $300 \mathrm{~K}$, (b) $5 \mathrm{~K}$.

prepared in an argon atmosphere which is $\sim 195 \mathrm{~K}$ ), which indicates their mean blocking temperatures. Furthermore, the relative sharpness of the ZFC curve peak for the sample prepared under vacuum and the fact that $T_{\mathrm{IRR}}$ and $T_{\mathrm{MEAN}}$ are quite close to each other $\left(T_{\text {IRR }} \sim 60 \mathrm{~K}\right)$ can be taken as an indication of a narrow size distribution for this sample. It is worth mentioning that the general distribution of blocking temperatures $\left(f\left(T_{\mathrm{B}}\right)\right)$ can be calculated as the temperature derivative of the difference between the $M_{\mathrm{FC}}$ and $M_{\mathrm{ZFC}}$ magnetizations $\left(\mathrm{d}\left[M_{\mathrm{FC}}-M_{\mathrm{ZFC}}\right]\right) / \mathrm{d} T$, allowing one to estimate correctly the mean blocking temperature $\left\langle T_{\mathrm{B}}\right\rangle .{ }^{19,20}$ The $\left\langle T_{\mathrm{B}}\right\rangle$ calculated from the distribution of blocking temperatures was found to be $\sim 56 \mathrm{~K}$ and $\sim 235 \mathrm{~K}$ (see Fig. $5 \mathrm{~b}$ ) for the sample prepared in a vacuum and in an argon atmosphere, respectively. From the $\left\langle T_{\mathrm{B}}\right\rangle$, we have calculated the mean size using the Néel relaxation model for isolated particles, i.e., $D=\left[6 k_{\mathrm{B}}\left\langle T_{\mathrm{B}}\right) \ln \left(t_{\mathrm{m}} f_{0}\right) / \pi K_{\text {eff }}\right]^{1 / 3}$, where $K_{\text {eff }}$ is the anisotropic energy of iron oxide, $t_{\mathrm{m}}$ the measurement time, $f_{0}$ is the frequency factor and $D$ is the size of iron oxide nanoparticles. Considering the value $2 \times$ $10^{4} \mathrm{erg} \mathrm{cm}^{-3}, 100 \mathrm{~s}$, and $10^{9} \mathrm{~s}^{-1}$ for $K_{\text {eff }}$ for cubic iron oxide $\left(\mathrm{Fe}_{3} \mathrm{O}_{4}\right.$ or $\left.\gamma-\mathrm{Fe}_{2} \mathrm{O}_{3}\right), t_{\mathrm{m}}$, and $f_{0}$, respectively, values of $9.2 \mathrm{~nm}$ (argon) and $5.6 \mathrm{~nm}$ (vacuum) are obtained for the iron oxide nanoparticles, which are in a good agreement with XRD and TEM findings.

The magnetization hysteresis loops were recorded at $300 \mathrm{~K}$ and $5 \mathrm{~K}$ in ZFC mode (Fig. 6). At $300 \mathrm{~K}$, the sample shows zero 
1 coercivity and retentivity, indicating that the particles are in the SPM state without saturation up to a field of $30 \mathrm{kOe}$, whereas at $5 \mathrm{~K}$, the coercivity is $\sim 345$ Oe and $\sim 165$ Oe for the sample prepared in an argon atmosphere and under vacuum condition, respectively. This can also be explained by the fact that $\gamma-\mathrm{Fe}_{2} \mathrm{O}_{3}$ is magnetically softer than $\mathrm{Fe}_{3} \mathrm{O}_{4}$. Additionally, coexisting phases could induce more magnetic frustration than the single magnetic phase, thereby inducing an increment of the coercivity for the sample prepared in an argon atmosphere against the

10 vacuum one. The saturation magnetization $\left(M_{\mathrm{S}}\right)$ of the sample prepared under vacuum is smaller than the one prepared under argon and bulk $\gamma-\mathrm{Fe}_{2} \mathrm{O}_{3}$. Roughly, this can be explained due to the fact the $M_{\mathrm{S}}$ value of bulk $\gamma-\mathrm{Fe}_{2} \mathrm{O}_{3}$ (76 emu g ${ }^{-1} @ 293 \mathrm{~K}$ ) is smaller than the value of $\mathrm{Fe}_{3} \mathrm{O}_{4},\left(93 \mathrm{emu} \mathrm{g}{ }^{-1} @ 290 \mathrm{~K}\right) .{ }^{21}$ Here we note that 15 nanoparticles prepared under vacuum are majority composed of $\gamma-\mathrm{Fe}_{2} \mathrm{O}_{3}$, whereas nanoparticles prepared under argon are of multi-phase composition with majority of $\gamma-\mathrm{Fe}_{2} \mathrm{O}_{3} / \mathrm{Fe}_{3} \mathrm{O}_{4}$. Reduced $M_{\mathrm{S}}$ compared to bulk values are commonly ascribed to spin canting or defects at the particle's outer surface. ${ }^{21}$ Indeed, previous work has identified iron oxide nanoparticles that can be composed of $\mathrm{Fe}_{3-\delta} \mathrm{O}_{4}$, for example, which is a solid solution of the end members $\mathrm{Fe}_{3} \mathrm{O}_{4}$ and $\gamma-\mathrm{Fe}_{2} \mathrm{O}_{3}$ (both ferrimagnets, FiM); $\mathrm{Fe}_{1-x} \mathrm{O}$ (wüstite) an antiferromagnet, AFM; or Fe, a ferromagnet, FM. Further, we have measured the hysteresis curves for both the samples at $5 \mathrm{~K}$ in a field cooled $\left(H_{\mathrm{FC}}=70 \mathrm{kOe}\right)$ mode for both the samples (figures not shown here) and observed that the sample prepared in an argon atmosphere displayed a measurable exchange bias of $\sim 40$ Oe at $5 \mathrm{~K}$, whereas the hysteresis curve for the sample prepared under vacuum is symmetric about the origin. This further confirms the presence of exchange coupling between the maghemite (ferromagnetic) and the wustite (antiferromagnetic) phase for the sample prepared in an argon atmosphere.

To have a deeper insight about these two samples, we have

35 further performed temperature dependence of dc magnetization after ZFC and FC at different magnetic fields. It is observed that the magnetization increases with increasing magnetic field, while the $T_{\mathrm{B}}$ shifts to lower temperatures with increasing magnetic field, indicating that the frozen spin glass state is 0 gradually destroyed under large magnetic fields for both samples (see Fig. 7 and 8). This coincides well with the bother SG systems. $^{22-25}$

We have further investigated the temperature dependence of the ac susceptibility measurements at different driving frequenthe range from $10 \mathrm{~Hz}-10 \mathrm{kHz}$ (see Fig $9 \mathrm{a}$ and b) for both samples prepared under argon flux or vacuum conditions. It is observed that $\chi^{\prime}(T)$ exhibits a strongly frequency dependent peak. As the frequency increases, the sharp peak shifts to higher temperatures, while the height decreases, suggesting a characteristic feature of typical SG behavior. The expected behaviour of blocking processes is evident in the plots, i.e. the occurrence of a maximum at $T_{\mathrm{B}}$, which shifted towards higher temperatures and decreased in height with increasing frequency. ${ }^{26}$ The effect that becomes visible upon sample cooling is directly related to the frequency dependence of $T_{\mathrm{B}}$ of the single-domain particles. Two key empirical relations are often
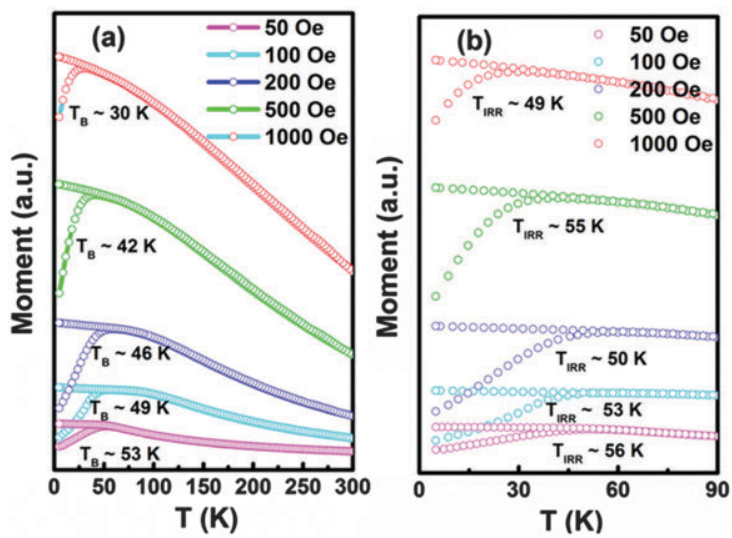

Fig. 7 (a) $T$-dependent magnetization $M(T)$ curves under FC and ZFC modes with different magnetic field magnetization curves measured under different magnetic fields up to $1 \mathrm{kOe}$, and (b) its enlarged view for the sample prepared under argon flux.
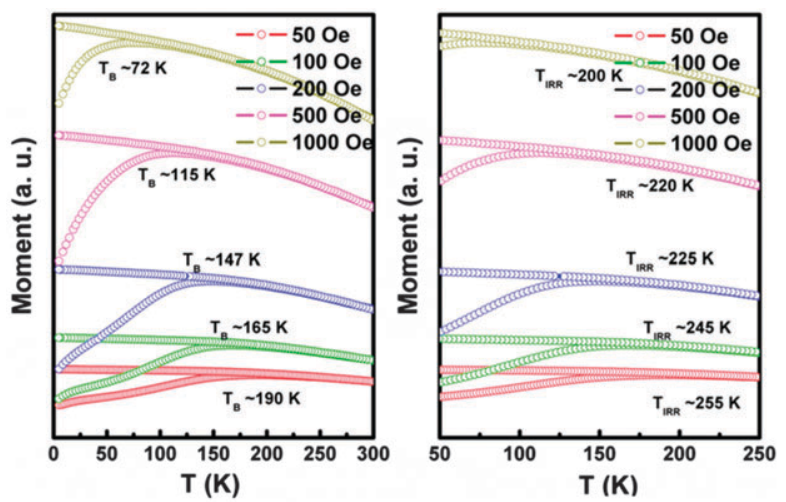

Fig. 8 (a) $T$-dependent magnetization $M(T)$ curves under $F C$ and ZFC modes with different magnetic field magnetization curves measured under different magnetic fields up to $1 \mathrm{kOe}$, and (b) its enlarged view for the sample prepared under vacuum conditions.

used as tools to compare the frequency dependence of $T_{\mathrm{B}}$ namely, $C_{1}=\Delta T_{\mathrm{B}} / T_{\mathrm{B}} \Delta \log _{10}(f)$, independent of any model and $C_{2}=\left(T_{\mathrm{B}}-T_{0}\right) / T_{\mathrm{B}}$, where $\Delta T_{\mathrm{B}}$ is the difference between the $T_{\mathrm{B}}$ measured in the $\Delta \log _{10}(f)$ frequency interval and $f$ represents the ac magnetic field frequency. ${ }^{26}$ The parameters $C_{1}, C_{2}$ and $T_{0}$ deliver a model-independent classification of the blocking/ freezing process. ${ }^{26-28}$

This law is phenomenological and without physical significance near $T_{0}$, and yet $C_{2}$ can be taken as an indicator to screen different $T_{\mathrm{B}}$ in closely related materials. Fig. 9c shows the variation of the $T_{\mathrm{B}}$ in the classical plot of $\log _{10}(\tau)$ vs. $1 / T_{\mathrm{B}}$ for both samples. For isolated nanoparticles, $f$-dependence of the $T_{\mathrm{B}}$ has been predicted, according to the SPM Neel model, to follow an Arrhenius law $\ln \left(\tau / \tau_{0}\right)=E_{\mathrm{B}} / k T_{\mathrm{B}}$, where $\tau=1 / f, \tau_{0}$ is the characteristic relaxation time constant $\left(10^{-9} \mathrm{~s}<\tau_{0}<10^{-12} \mathrm{~s}\right)$, and $E_{\mathrm{B}}$ is the energy barrier of the NPs for the moment reversal. ${ }^{27}$ Thus, $E_{\mathrm{B}}=K V$, and $K$ represents the effective anisotropy constant and $V$ the volume of the particle. The terms $E_{\mathrm{B}}$ and $K$ can be estimated from analyses of the experimental data. 

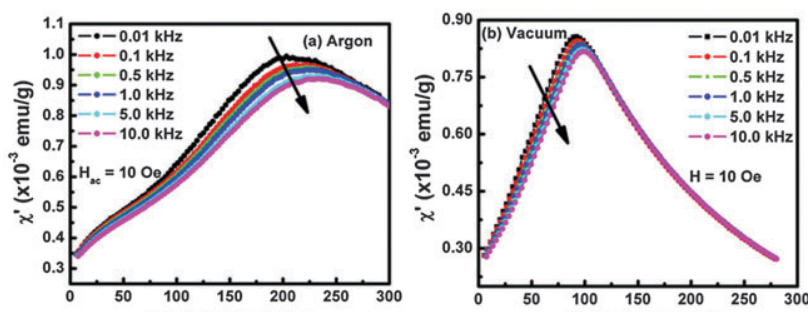

- Vacuum $E_{8} / k_{8} 7976 K$ Fit: Arrhenius law
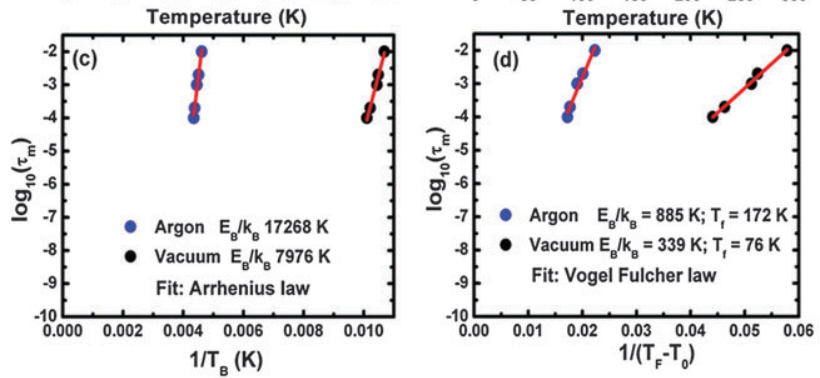

Fig. 9 ( $a$ and b) T-dependence of the real part of the ac susceptibility for the prepared under argon and vacuum, respectively, at different frequencies. Arrow indicates increasing frequencies. ( $c$ and $d$ ) variation of $T_{B}$ in the classical Arrhenius and Vogel-Fulcher plot of $\log _{10}(\tau)$ vs. 1/T.

Even though the fitted straight-lines in Fig. (9c) seem to well support the experimental findings; the fitted parameters obtained had no physical meaning for both the samples. Particularly, the $\tau_{0}$ values in both samples were found to be much smaller than those physically accepted, and $E_{\mathrm{B}}$ values were found to be too high as compared to the reference values of $E_{\mathrm{B}}=8440 \mathrm{~K}$ for iron oxide (calculated using $K=5.5 \times 10^{6} \mathrm{erg}$ $\left.\mathrm{cm}^{-3}\right)$. Thus, the experimentally observed variations in $\chi^{\prime}(T)$ for these samples were not consistent with the simple superparamagnetic blocking behaviour of independent particles. The data were then analysed by using the Vogel-Fulcher relation ${ }^{26}$ and can be written as $\tau=\tau_{0} \exp \left\{E_{\mathrm{A}} / k_{\mathrm{B}}\left(T_{\mathrm{B}}-T_{0}\right)\right\}$. Here $T_{0}$ is an effective temperature with a similar origin to that used to reproduce the dc susceptibility in the superparamagnetic regime and $T_{\mathrm{B}}$ is the characteristic temperature signaling the onset of the blocking process.

Taking into account the Vogel-Fulcher relation, the calculated fitted parameters are given in Fig. 9d, and can be compared with the values extracted by using the Néel-Arrhenius relation. The Vogel-Fulcher law provides more reasonable values of $\tau_{0}$ and $E_{\mathrm{B}}$ for both samples, comparable to those observed for spin-glass (SG) systems. ${ }^{26}$ For sample prepared temperature is enhanced by more than two times in comparison to vacuum one, $\left(T_{0}=76 \mathrm{~K}\right.$ for vacuum, $T_{0}=172 \mathrm{~K}$ for argon). In this case, the VG fitting gives the following values of the parameters: $\tau_{0}=1.0 \times 10^{-10} \mathrm{~s}, T_{0}=76 \mathrm{~K}$ and $\tau_{0}=5.0 \times$ $10^{-12} \mathrm{~s}$, and $T_{0}=172 \mathrm{~K}$, respectively, for the sample prepared in a vacuum and in an argon atmosphere, respectively. A good agreement of the experimental data and the Vogel-Fulcher (VG) law evidences that the phenomenon taking place at maximum of susceptibility is related to blocking of an assembly of interacting particles rather than a collective freezing as that occurring in a spin-glass system.

\section{Conclusions}

Synthesis methods clearly show the effect of argon and vacuum environments on the structure-property relationship of iron oxide nanoparticles. Detailed XRD, XANES and Mössbauer experiments suggest that the dominating chemical phase is $\gamma-\mathrm{Fe}_{2} \mathrm{O}_{3}$ for both the samples. Vacuum conditions result in a narrow particle size distribution as compared to the argon atmosphere. DC magnetization measurements revealed that nanoparticles are superparamagnetic above the blocking temperature. However, the analysis of ac susceptibility data shows that magnetic dynamics of these nanoparticles is strongly influenced by spin-glass features and is well described by the Vogel-Fulcher (VG) law for interacting superparamagnetic particles. On the other hand, endeavor to fit the data with the Neel-Brown (NA) model for thermally non-interacting superparamagnetic (SPM) particles is unsuccessful and yields an unphysical small value of relaxation time constant $\tau_{0}$.

\section{Acknowledgements}

SKS is grateful to FAPEMA for providing financial support. Sarveena would like to thank UGC-DAE CSR, Indore Centre for financial support under the CRS Scheme. JM would like to acknowledge the full support by Conicet. DKS is thankful to DST-India, New Delhi for the support through DST-Inspire faculty scheme. Mr Rakesh Sah is thankfully acknowledged for help during XAS measurements.

\section{References}

1 B. Z. Zeng and S. Sun, Adv. Funct. Mater., 2008, 18, 391.

2 Y. Hou, Z. Xu and S. Sun, Angew. Chem., Int. Ed., 2007, 46, 6329.

3 C. J. Chen, R. K. Chiang, H. Y. Lai and C. R. Lin, J. Phys. Chem. C, 2010, 114, 4258.

4 Y. S. Dedkov, U. Rudiger and G. Guntherodt, Phys. Rev. B: Condens. Matter Mater. Phys., 2002, 65, 064417.

5 P. Seneor, A. Fert, J. L. Maurice, F. Montaigne, F. Petroff and A. Vaures, Appl. Phys. Lett., 1999, 74, 4017.

6 A. K. Gupta and S. Wells, IEEE Trans. Nanobiosci., 2004, 3,66 .

7 S. W. Charles, in Magneticfluids (ferrofluids), ed. J. L. Dormann and D. Fiorani, Magnetic properties of fine particles, Elsevier, North Holland, 1992, pp. 267-374.

8 A. K. Gupta and A. S. G. Curtis, Biomaterials, 2004, 25, 3029.

9 S. K. Sharma, J. M. Vargas, N. M. Vargas, S. C. Sepúlveda, D. Altbir, K. R. Pirota, R. Zboril, G. Zoppellaro and M. Knobel, RSC Adv., 2015, 5, 17117.

10 S. K. Sharma, J. M. Vargas, S. Kumar, C. G. Lee, K. R. Pirota and M. Knobel, J. Alloys Compd., 2011, 509, 6414.

11 W. Wu, Z. Wu, T. Yu, C. Jiang and W. S. Kim, Sci. Technol. Adv. Mater., 2015, 16, 023501.

12 R. A. Young, A. Sakthivel, T. S. Moss and C. O. P. Santos, J. Appl. Crystallogr., 1995, 28, 366. 
113 L. Bleicher, J. M. Sasaki and C. O. P. Santos, J. Appl. Crystallogr., 2000, 33, 1189.

14 C. T. Meneses, W. H. Flores, F. Garcia and J. M. Sasaki, J. Nanopart. Res., 2006, 9, 501.

515 R. A. Young, The Rietveld Method, Oxford Science Publications, New York, 1993, p. 126.

16 F. M. F. de Groot, M. Grioni, J. C. Fuggle, J. Ghijsen, G. A. Sawatzky and H. Petersen, Phys. Rev. B: Condens. Matter Mater. Phys., 1989, 40, 5715.

17 W.-C. Wang, S.-Y. Chen, P.-A. Glans, J. Guo, R.-J. Chen, K.-W. Fong, C.-L. Chen, A. Gloter, C.-L. Chang, T.-S. Chan, J.-M. Chen, J.-F. Lee and C.-L. Dong, Phys. Chem. Chem. Phys., 2013, 15, 14701.

18 R. M. Cornell and U. Schwertmann, The Iron Oxides: Structure, Properties, Reactions, Occurrence and Uses, Wiley VCH, Weinheim, Germany, 2003; J. A. Ramos Guivar, A. Bustamante, J. Flores, M. Mejía Santillan, A. M. Osorio, A. I. Martínez, L. De Los Santos Valladares and C. H. W. Barnes, Hyperfine Interact., 2014, 224, 89.

19 S. Barzilai, Y. Goldstein, I. Balberg and J. S. Helman, Phys. Rev. B: Condens. Matter Mater. Phys., 1981, 23, 1809.
20 J. C. Denardin, A. L. Brandl, M. Knobel, P. Panissod, A. B. Pakhomov, H. Liu and X. X. Zhang, Phys. Rev. B: Condens. Matter Mater. Phys., 2002, 65, 064422.

21 E. Wetterskog, C. W. Tai, J. Grins, L. Bergstrom and G. S. Alvarez, ACS Nano, 2013, 7(8), 7132.

22 B. Song, J. K. Jian, H. Q. Bao, M. Lei, H. Li, G. Wang, Y. P. Xu and X. L. Chen, Appl. Phys. Lett., 2008, 92, 192511.

23 B. S. Wang, P. Tong, Y. P. Sun, X. B. Zhu, Z. R. Yang, W. H. Song and J. M. Dai, Appl. Phys. Lett., 2010, 97, 042508.

24 X. H. Zhang, Q. Yuan, J. C. Han, J. G. Zhao, J. K. Jian, Z. H. Zhang and B. Song, Appl. Phys. Lett., 2013, 103, 022405.

25 S. K. Sharma, R. Kumar, S. Kumar, M. Knobel, V. V. Siva Kumar, V. R. Reddy, M. Singh and C. G. Lee, J. Phys.: Condens. Matter, 2008, 20, 235214.

26 J. M. Vargas, A. Srivastava, A. Yourdkhani, L. Zaldivar, G. Caruntu and L. Spinu, J. Appl. Phys., 2011, 110, 064304.

27 M. Knobel, W. C. Nunes, L. M. Socolovsky, E. De. Biasi, J. M. Vargas and J. C. Denardin, J. Nanosci. Nanotechnol., 2008, 8, 2836.

28 J. L. Dormann, L. Bessais and D. Fiorani, J. Phys. C: Solid State Phys., 1988, 21, 2015. 